\title{
AN INVESTIGATION OF ON-DUTY INJURIES IN INDUSTRY AND THEIR RELATION TO THE WORKING SITUATION ${ }^{\dagger}$
}

\author{
R. W. TWORECK*
}

In present-day industry, with its complex machinery, and in certain areas. hard physical work, injuries and strains are commonplace among workers. This leads to a loss of manhours, production loss, compensation requests and in the long run an "unhealthy" working climate. To prevent these injuries and strains would be beneficial for all concerned.

The function of a physiotherapist in industry encompasses four areas: preventative, curative, rehabilitative and educative care. Curing on the spot could mean further reduction of loss of man-hours. Over one-half of all industrial injuries are directly related to posture and bodymechanics and the incidence is still increasing particularly with regard to back injuries (statistical records of the National Occupational Safety Association).

This project was aimed at investigating the incidence and types of short-term, musculo-skeletal injuries which were sustained between May 1981 and May 1982 in a specific South African industry. It was further hoped to determine whether physiotherapists could fulfill a useful role in the ergonomic aspect in this field and if so, further research in this area would be indicated.

A study was conducted at a factory responsible for packaging and labelling in the Cape Peninsula. This industry is divided into sections, different types of work being done in each section. The staff consisted of 883 workers of all race groups. The information on work injuries was obtained from the personnel files in the surgery. Persons who had suffered from short-term musculo-skeletal injuries were interviewed to determine the mechanism of injury. At the same time the workers were observed for faults in postural habits and kinetic handling. In the table the following are recorded:

- the departments with the total number of people working there.

- the number of injured cases of the selected group in each department.

- the number of injuries in each department.

These statistics demonstrate relatively serious short-term musculo-skeletal injuries (i.e. serious enough to seek medical help) suffered in the course of the year. Of the 883 workers in the factory $19.25 \%$ were affected.

A ccording to the ratio between the number of injuries and number of people in each department, the four departments with the highest accident rate were then selected for closer investigation. These departments were:

- Lithography

- Binding

- Sorting

- Breaking out.

Table 1

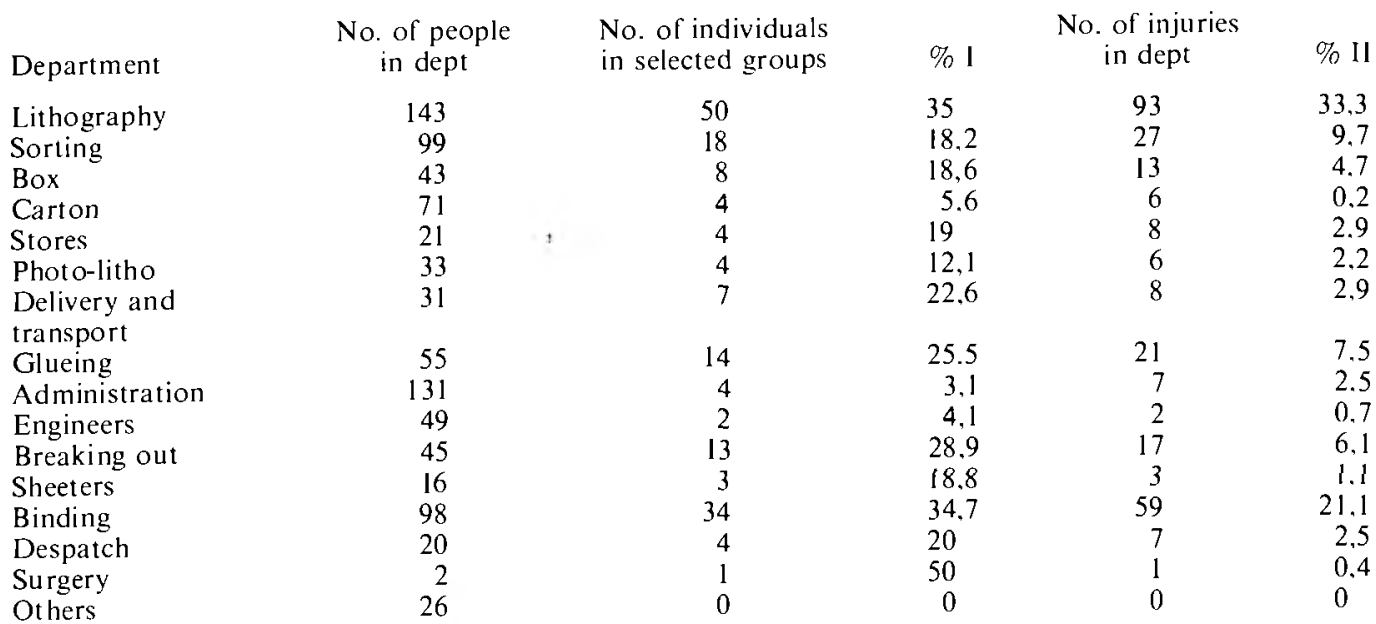

$\%$ calculated as follows:

I No. of individuals in selected group $\mathrm{x} 100$

No. of people in department

II No. of injuries in department $\times 100$

Total no. of injuries

† Abstract of project completed as course requirement.

* Final year B.Sc. (Physiotherapy) student at the University of Stellenbsch.

Received 30 March 1983. 
Table 2

\author{
Diagnoses \\ Lumbar back pain \\ Shoulder injury \\ Hand injury \\ Neck pain \\ Thoracic pain \\ Knee injury \\ Muscle injury \\ Ankle injury
}

Frequency
239
17
8
5
5
2
2
1

The types of musculo-skeletal injuries which occurred in the timespan of one year are listed in Table 2 . They ranged from low back pain to ankle injuries.

The highest $\%$ of the total number of injuries was low back pain (LBP), 85,7\%. The data in Table 2 also show that this injury had a significant total loss of 581 working days compared to shoulder injuries with a total day loss of 25 working days.

Table 3.

Mechanism

Back and hip bending with knees straight

Turn while coming upright

Leaning forward

Lifting, hammering and bending

Standing

Pulling

Falling backwards

Bending forwards

Contact

Pushing

Sitting and turning

Unknown Total

$\begin{array}{cc}\text { No. of injury } & \begin{array}{c}\text { \% of total } \\ \text { no. of back } \\ \text { injuries }\end{array} \\ 147 & 61,8 \\ 25 & 10.5 \\ 21 & 8.8 \\ 14 & 5,9 \\ 5 & 2,1 \\ 4 & 1,7 \\ 4 & 1,7 \\ 4 & 1,7 \\ 1 & 0.4 \\ 1 & 0,4 \\ 1 & 0.4 \\ 5 & 2,1\end{array}$

$\begin{array}{cr}\text { Mean day loss } & \text { Total da loss } \\ 2,4 & 581 \\ 1,5 & 25 \\ 0 & 0 \\ 3,8 & 19 \\ 2 & 10 \\ 2,5 & 5 \\ 0 & 0 \\ 0 & 0\end{array}$

The mechanisms of injury, resulting in LBP were evaluated (Table 3 ).

It is obvious that most injuries were the result of poor kinetic handling.

This is by no means a complete record of all the back problems in the factory. Several workers accepted their back pain as a minor problem and normal occurrence, and did not think it necessary to seek medical help and advice.

Of the short term musculo-skeletal injuries LBP was the most significant, according to the number of injurics (293) and total loss of working days (581). This could be attributed to poor kinetic habits, which included wrong methods of bending, and lifting, sitting and standing for long uninterrupted periods of time. The combination of heavy physical work and an increase in work load during seasonal peaks showed a high increase in accident rate which adversely affected the work load to be done. This means that the remaining workers had more work to cover. This aga in could cause increased stress to the limits of workers' capacity, leading to more injuries. This vicious cycle results in decreased productivity during peak seasons.

Application of ergonomics is justified by the high incidence of back injuries. The physiotherapist's knowledge could enable her to give advice to engineers and workers. It would be advisable to make management and workers more awa re of the actual magnitude of the problem and stimulate further efforts to ensure the safety and health of workers.

\section{MOBILISATION SLIDE-TAPE PROGRAMMES}

Seventeen slide-tape programmes on Mobilisation of Joints by Gregory Grieve, recorded on standard audiotape cassettes and illustrated by full-frame $35 \mathrm{~mm}$ transparencies.

For further details contact:

Graves Medical Audiovisual Library, Holly House, 220 New London Road, Chelmsford, Essex, CM 2 9BJ, UK. 